\title{
The usefulness of contrast during exercise echocardiography for the assessment of systolic pulmonary pressure
} Luis R Lopes*1, Maria J Loureiro1, Rita Miranda1, Sofia Almeida1, Ana R Almeida ${ }^{1}$, Ana Cordeiro ${ }^{2}$, Carlos Cotrim $^{1}{ }^{\text {and Manuel Carrageta }}{ }^{1}$

\author{
Address: ${ }^{1}$ Cardiology Department, Hospital Garcia de Orta, Av. Torrado da Silva, Pragal 2801-951 Almada, Portugal and ${ }^{2}$ Reumatology \\ Department, Hospital Garcia de Orta, Av. Torrado da Silva, Pragal 2801-951 Almada, Portugal \\ Email: Luis R Lopes* - luisrlopes@hotmail.com; Maria J Loureiro - loureiro.mj@netcabo.pt; Rita Miranda - ritasmiranda@gmail.com; \\ Sofia Almeida - sofia.cardio@gmail.com; Ana R Almeida - anaalmeida@portugalmail.pt; Ana Cordeiro - cordeiro.ana@gmail.com; \\ Carlos Cotrim - carlosadcotrim@hotmail.com; Manuel Carrageta - mcarrageta@mail.telepac.pt \\ * Corresponding author
}

Published: 13 October 2008

Cardiovascular Ultrasound 2008, 6:5 I doi:|0.| |86/|476-7|20-6-5|
Received: 28 August 2008

Accepted: 13 October 2008

This article is available from: http://www.cardiovascularultrasound.com/content/6/I/5 I

(c) 2008 Lopes et al; licensee BioMed Central Ltd.

This is an Open Access article distributed under the terms of the Creative Commons Attribution License (http://creativecommons.org/licenses/by/2.0), which permits unrestricted use, distribution, and reproduction in any medium, provided the original work is properly cited.

\begin{abstract}
Background: The systolic pulmonary artery pressure (PAPs) can be accurately estimated, noninvasively, using continuous-wave Doppler (CWD) ultrasound measurement of the peak velocity of a tricuspid regurgitant (TR) jet.

However, it is often difficult to obtain adequate tricuspid regurgitation signals for measurement of PAPs, what could lead to its underestimation. Therefore, utilization of air-blood-saline contrast has been implemented for the improvement of Doppler signal in several clinical contexts.

It is now recommended in the evaluation of patients with pulmonary hypertension. Physical activity is severely restricted in patients with PAH, being exertional dypnea the most typical symptom. Exercise stress echo-Doppler imaging allows assessment of the response to exercise. It is an excellent screening test for patients with suspected PAH. Our purpose was to evaluate the value and accuracy of agitated saline with blood contrast echocardiography, in the improvement of the Doppler signal, to quantify PAPs during treadmill exercise-echocardiography.

Purpose: To evaluate the value of contrast echocardiography, using agitated saline with blood, in the improvement of the Doppler signal used to quantify the pulmonary artery systolic pressure during exercise.

Methods: From a total of $4 \mathrm{I}$ patients (pts), we studied 38 pts (93\%), 35 women, aged $54 \pm 12$ years-old. 27 with the diagnosis of systemic sclerosis, 10 with history of pulmonary embolism and one patient with a suspected idiopathic $\mathrm{PAH}$, who were referred to the Unity of Heart Failure and Pulmonary Hypertension for screening of PAH. According to the Unity protocol, a transthoracic echocardiogram was made, in left decubitus (LD), with evaluation of right ventricle-right atria gradient (RV/RAg). A peripheral venous access was obtained, with a 3-way stopcock and the patients were placed in orthostatism (O), with a new evaluation of RV/RAg. Exercise echocardiography (EE) was begun, with evaluation of RV/RAg at peak exercise $(P)$ and afterwards agitated saline ( $8 \mathrm{cc}$ with I $\mathrm{cc}$ of air and I $\mathrm{cc}$ of blood) was injected, followed by a new evaluation of RV/RAg (PC) and then the interruption of the EE. Pulmonary Hypertension was diagnosed when $\mathrm{RV} / \mathrm{RAg}$ at the end of the exercise was superior to $40 \mathrm{mmHg}$.
\end{abstract}


Results: The quality of Doppler signal was deteriorated in 5 pts, maintained in 6 pts and improved in 26 pts, with the use of contrast. In one patient, an interventricular septal defect was diagnosed. In 6 pts, a Doppler signal was only obtained with the use of contrast. In I 5 pts, a RV/RAg superior to $40 \mathrm{mmHg}$ was only obtained with the use of contrast. Of these, 9 have already been submitted to right heart cathetherism, that confirmed the diagnosis of pulmonary hypertension in 5 of them (56\%). RV/RAg (P) was $44 \pm \mathrm{II} \mathrm{mmHg}$ and $\mathrm{RV} / \mathrm{RAg}(\mathrm{PC})$ was $54 \pm \mathrm{II} \mathrm{mmHg}, \mathrm{p}<0,00 \mathrm{I}$.

Conclusion: I. The method is applicable in a large number of patients. 2. RV/RA gradients obtained at peak exercise are higher with the use of contrast, and the clinical meaning of this difference should be evaluated in a larger number of pts submitted to right heart cathetherism. The high number of false positives should lead to a higher diagnostic threshold. 3 . This method seems to have relevant clinical value in the diagnosis of pulmonary arterial hypertension.

\section{Introduction}

The systolic pulmonary artery pressure (PAPs) can be accurately estimated, non-invasively, using continuouswave Doppler (CWD) ultrasound measurement of the peak velocity of a tricuspid regurgitant (TR) jet. The systolic pressure gradient (delta $\mathrm{P}$ ) between right ventricle and right atrium is calculated by the modified Bernoulli equation (delta $\mathrm{P}=4 \mathrm{~V} 2$ ). Adding the transtricuspid gradient to the mean right atrial pressure - estimated by the assessment of the righ atrial size, inferior vena cava diameter and inferior vena cava inspiratory collapse - gives predictions of right ventricular systolic pressure that correlated well with right heart catheterization values, in several studies made in multiple clinical contexts, both in congenital and acquired heart disease [1-5].

Noninvasive estimation of pulmonary arterial pressure is important for haemodynamic monitoring of patients with heart disease, obviating the need for repeated catheterization.

However, it is often difficult to obtain adequate tricuspid regurgitation signals for measurement of PAPs, what could lead to its underestimation. Therefore, utilization of air-blood-saline contrast has been implemented for the improvement of Doppler signal in several clinical contexts. It is now recommended in the evaluation of patients with pulmonary hypertension [6-8].

Physical activity is severely restricted in patients with $\mathrm{PAH}$, being exertional dypnea the most typical symptom. The parameters obtained at rest poorly correlate with symptoms or exercise response and have modest prognosis significance, in contrast with the 6 minute walking test or cardiopulmonary exercise test, that assess exercise intolerance.

Exercise stress echo-Doppler imaging allows assessment of the response to exercise of left and right ventricular function, transvalvular and prosthetic valve gradients, and right ventricular systolic pressure. It has substantial clini- cal use in the evaluation of patients with equivocal evidence of mitral stenosis, suspition of dynamic mitral insufficiency or prosthetic valve dysfunction, aortic stenosis and impaired left ventricular systolic function, diseases associated with pulmonary hypertension or cor pulmonale, and among patients with dyspnea of unknown origin [9].

It is an excellent screening test for patients with suspected PAH: in asymptomatic individuals at risk it may unmask abnormal increases in pulmonary artery pressures during exercise, corresponding to early stage disease $[9,10]$.

It was already demonstrated that exercise echocardiography can be used to determine the extent of pulmonary vascular damage in patients with chronic obstructive pulmonary disease, by showing the presence of exertional pulmonary hypertension in subjects with normal PAPs at rest [11].

It is also useful in patients with connective tissue disease to screen for lung involvement by identifying exertional pulmonary hypertension, and in patients with established pulmonary hypertension to choose and monitor the effects of therapeutic interventions on the PAPs [11].

We believe that treadmill exercise is more closely related, compared with bycicle exercise, to the daily physical activity demands. Although the feasibility of treadmill stress echocardiography is described as lower in most of the publications, there isn't, at the moment, any study that makes a direct comparison between the two types of exercise stress echocardiography.

Our purpose was to evaluate the value and accuracy of agitated saline with blood contrast echocardiography, in the improvement of the Doppler signal, to quantify PAPs during treadmill exercise-echocardiography. 


\section{Methods \\ Population}

From 41 patients, we studied 38 patients (93\%) - 35 women $(92 \%)$ - aged $54 \pm 11$ years-old. Three patients were excluded due to poor quality images. Twenty-seven patients had a diagnosis of systemic sclerosis, 10 patients had pulmonary embolism history and one patient had a suspected idiopathic pulmonary hypertension (PAH) without TR. They were referred to the Unity of Heart Failure and Pulmonary Hypertension for screening of PAH.

\section{Exercise echocardiography}

A Philips Sonos $7500{ }^{\circledR}$ echocardiograph was used. All exams were digitally recorded.

According to the Unity protocol, a rest transthoracic echocardiogram was made first, in left decubitus (LD), which included evaluation of right ventricle-right atria gradient (RV/RAg). A peripheral venous access was then obtained, with a 3-way stopcock, and the patients were placed in orthostatism (O), with a new evaluation of RV/ RAg.

Exercise echocardiography (EE), performed according to the European Association of Echocardiography guidelines [12], was begun (additional file 1), with evaluation of RV/ RAg at peak exercise (P) and afterwards agitated saline contrast (8 cc with $1 \mathrm{cc}$ of air and $1 \mathrm{cc}$ of blood) was injected, followed by a new evaluation of RV/RAg (PC) still in orthostatism- and the interruption of the EE (additional files 2 and 3). Pulmonary Hypertension was diagnosed when RV/RAg at the end of the exercise was superior to $40 \mathrm{mmHg}$ (according to previous reports on normal values of PAPs during exercise in healthy individuals).

Heart rate, systemic blood pressure and EKG were monitored throughout the exam.

\section{Right heart catheterization}

The patients considered to have PAH by echocardiography were submitted to right heart catheterization, with a Swan-Ganz catheter.

According to the published guidelines, PAH was defined by a mean PAP > $25 \mathrm{mmHg}$ at rest or $>30 \mathrm{mmHg}$ with exercise [13].

\section{Statistical analysis}

We compared the value of peak exercise RV/RAg, before and after injection of contrast, with a student $t$ test.

Of the patients with echocardiographically diagnosed $\mathrm{PAH}$, we compared the results of the non-invasive and invasive evaluation.
We also analyzed and described the number of patients with improved, equal and worsened CWD signal of the TR jet, after injection of contrast.

\section{Results}

The general echocardiographic parameters of the population are characterized in table 1.

The quality of Doppler signal was improved in twenty-six patients, deteriorated in five patients and maintained in six patients, with the use of contrast (Figure 1, Figure 2 and Figure 3).

In one patient, a ventricular septal defect was diagnosed (additional file 4).

In six patients, a Doppler signal was only obtained with the use of contrast.

In fifteen patients, a RV/RAg superior to $40 \mathrm{mmHg}$ was only obtained with the use of contrast. Of these, nine have already been submitted to right heart cathetherization, that confirmed the diagnosis of pulmonary hypertension in five of them $(56 \%)$.

The mean value of RV/RA gradients at peak exercise, without contrast, was $44 \pm 11 \mathrm{mmHg}$ and after contrast administration was $54 \pm 11 \mathrm{mmHg}, \mathrm{p}=0,001$. (Figure 4).

Table I: General echocardiographic characterization of the population

Echocardiographic parameters

Left ventricle end-diastolic diameter (M-mode $-\mathrm{mm}) \quad 47 \pm 4,9$

\begin{tabular}{ll}
\hline Fractional shortening (\%) & $38 \pm 7,2$
\end{tabular}

Interventricular septum thickness (mm) $\quad 8,9 \pm 1,4$

\begin{tabular}{ll}
\hline Posterior wall thickness $(\mathrm{mm})$ & $8 \pm 1,5$
\end{tabular}

Right ventricle area (mm2) $\quad 9,94 \pm 2,56$

\begin{tabular}{ll}
\hline Left ventricle area $(\mathrm{cm} 2)$ & $21 \pm 5$, ,
\end{tabular}

Right atria area $(\mathrm{cm} 2) \quad 9,47 \pm 3,1$

$\begin{array}{ll}\text { Left atria area }(\mathrm{cm} 2) & 12,64 \pm 3,2\end{array}$

Tricuspid annular plane systolic excursion ( $\mathrm{mm}$ )

$22 \pm 3,7$

Inferior vena cava diameter $(\mathrm{mm})$

$15,8 \pm 3,6$ 


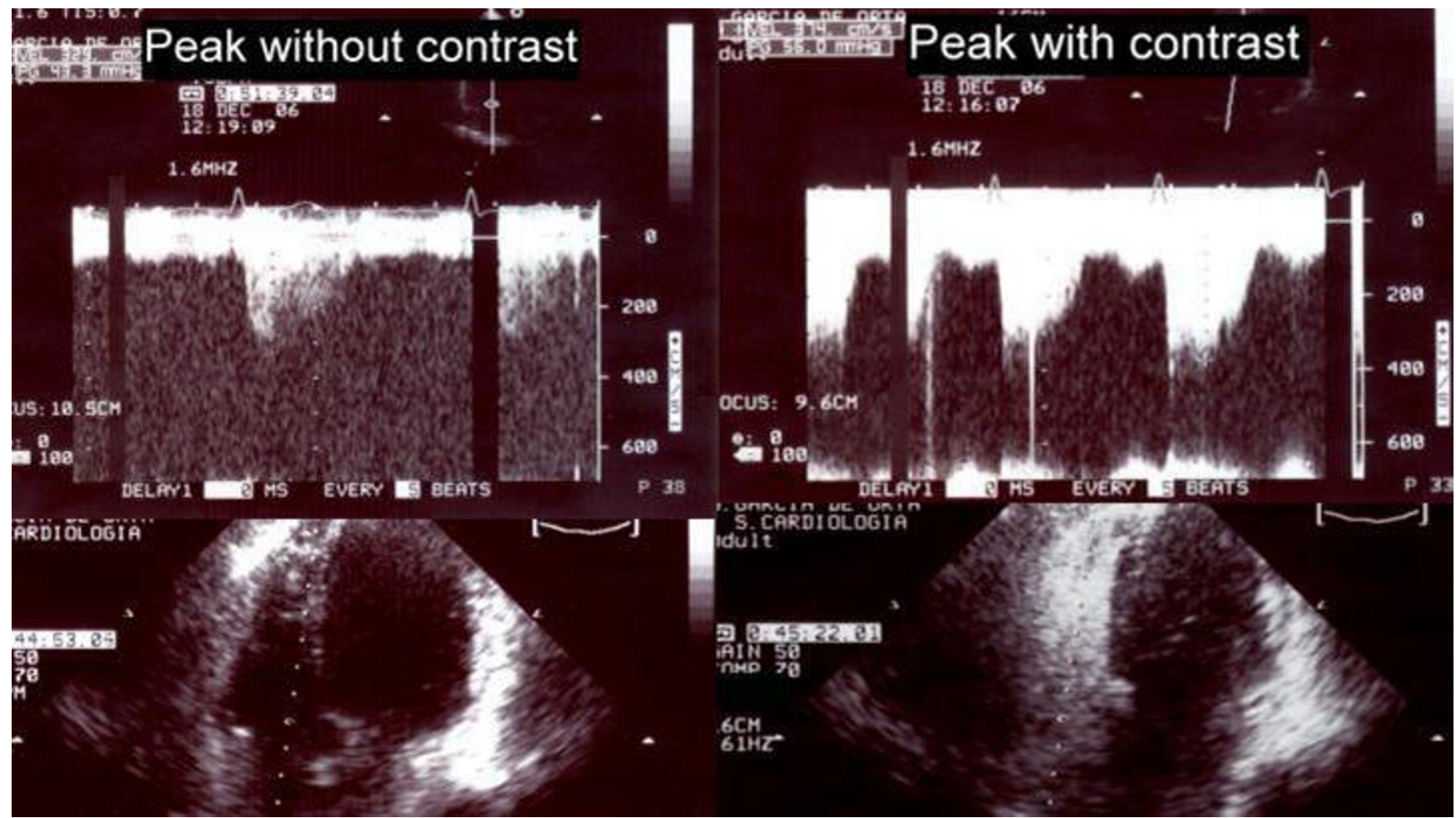

Figure I

Continuous wave Doppler signal of the tricuspid regurgitant jet at peak exercise, before and after the injection of contrast.

\section{Discussion and conclusion}

This method is feasible and applicable in a large number of patients, which is an important characteristic for its use as a screening test.

As occurred in previously published studies, the use of contrast improved the tricuspid regurgitation jet Doppler signal in the vast majority of patients. In some of them, a signal was only obtained after the administration of contrast.

Furthermore, RV/RA gradients obtained at peak exercise are significantly higher with the use of contrast. In the patients with RV/RA gradients superior to $40 \mathrm{mmHg}$ only after the administration of contrast, there seems to be a significant number of false positives.

The number of false positives can possibly be diminished by the use of a lower cut-off value, but the meaning of the difference between peak RV/RA gradients before and after administration of contrast should be evaluated in a higher number of patients submitted to right hear catheterization.
One of the limitations of the method, at this point, is the absence of a clear definition of threshold values.

In conclusion, treadmill exercise echocardiography with contrast seems to have relevant clinical value in the screening and diagnosis of pulmonary arterial hypertension.

Furthermore, it is once again demonstrated that a low cost "home-made" contrast can have a high diagnostic yield, with security advantages when compared to other contrast more expensive and less safe (FDA black box warning on ultrasound contrast media).

The utilization of contrast should probably be limited to the patients with very poor tricuspid regurgitation jet signal, to obviate the apparently high number of false positives results. The augmentation of Doppler signal with contrast, in patients that already have a good quality one, can generate significantly higher RV/RA gradients, including values superior to $40 \mathrm{mmHg}$, without a clear clinical meaning. So, we think that contrast should be an aid to obtain a measurable RV/RA gradient, but should not be used routinely in all patients submitted to this exam. 


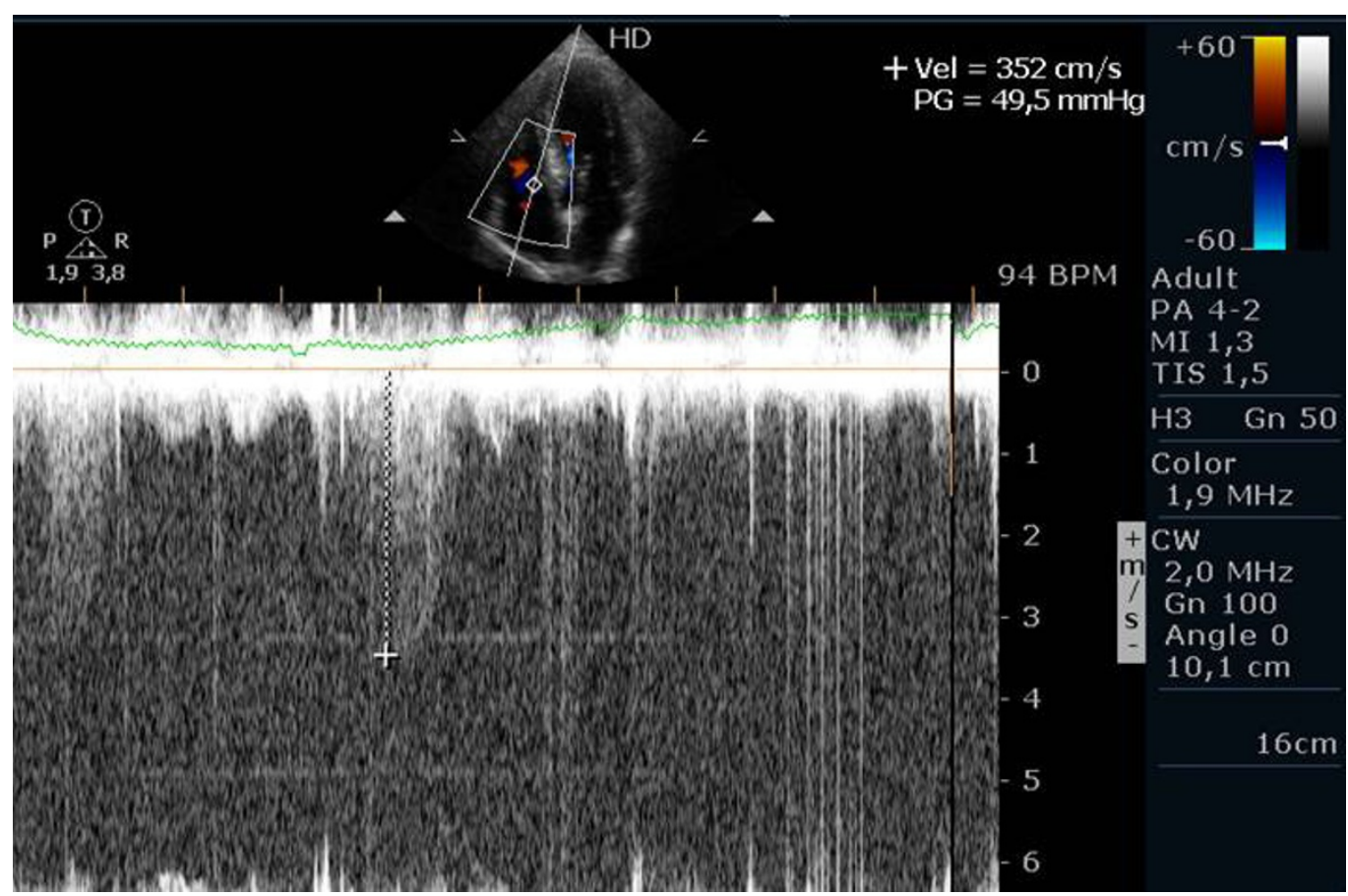

Figure 2

Continuous wave Doppler signal of the tricuspid regurgitant jet at peak exercise, before the injection of contrast.

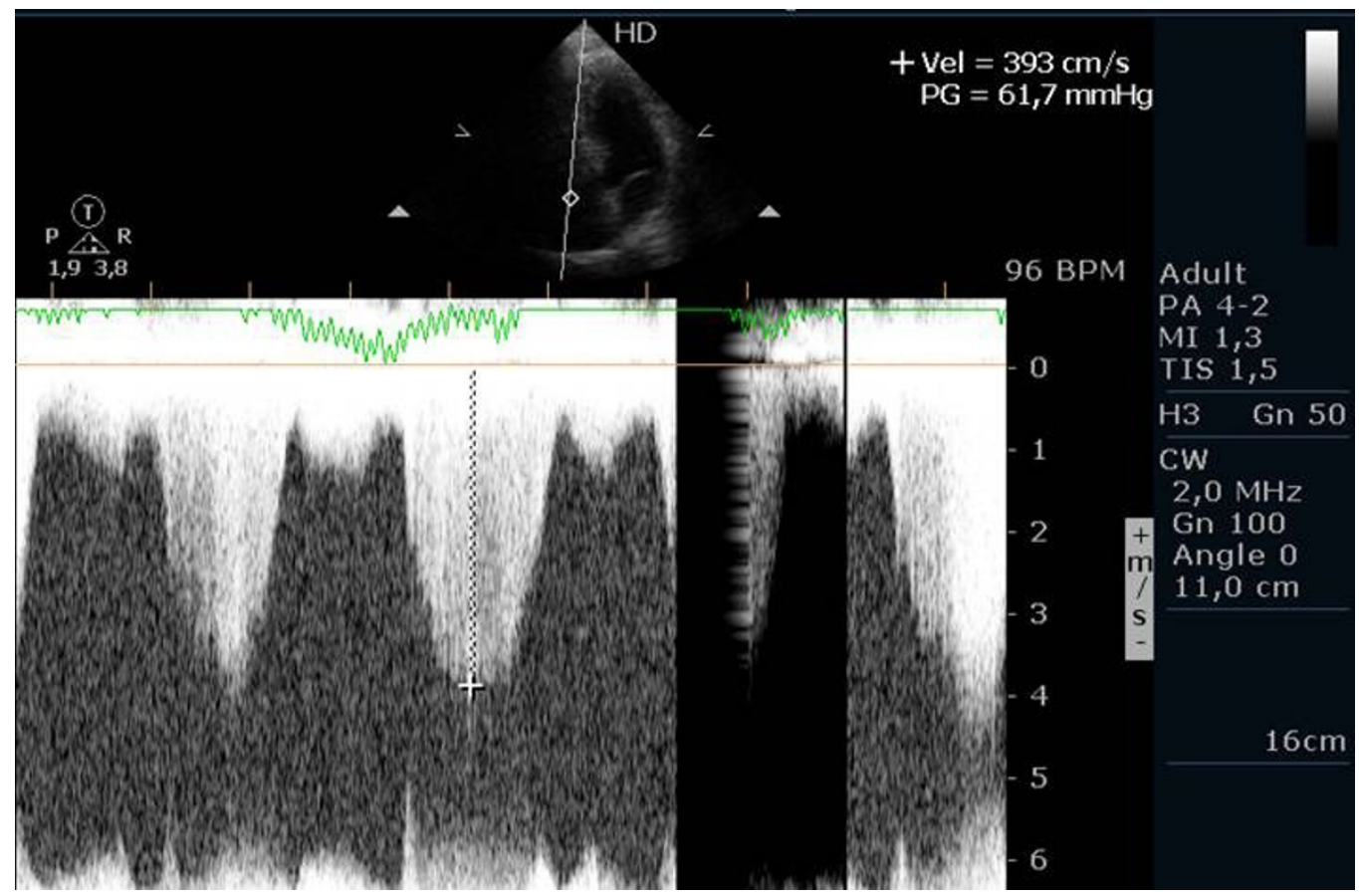

Figure 3

Continuous wave Doppler signal of the tricuspid regurgitant jet at peak exercise, after the injection of contrast. 


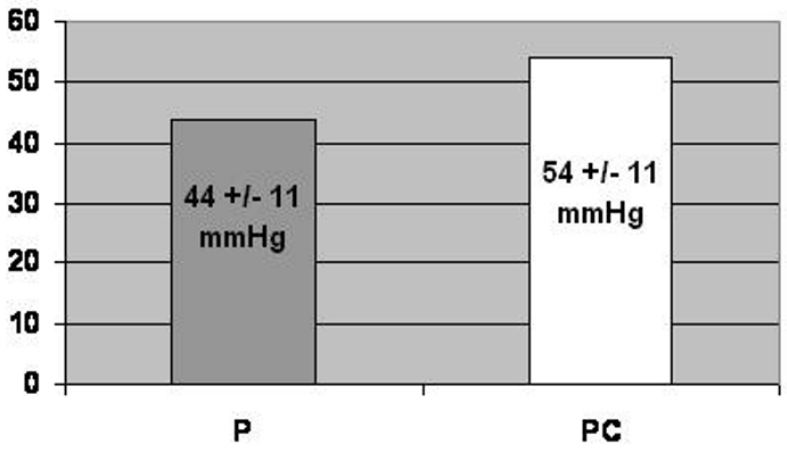

Figure 4

Comparison between RV/RA gradients at peak exercise, without contrast $(P)$, and after contrast administration (PC).

\section{Competing interests}

The authors declare that they have no competing interests.

\section{Authors' contributions}

LRL performed exercise echocardiography, reviewed literature and wrote the manuscript. CC performed exercise echocardiography, made clinical assessment of the patients, participate in drafting, and revised the manuscript for important intellectual content. MJL, RM, SA, ARA were responsible for clinical assessment of the patients and revised the manuscript for important intellectual content. AC was responsible for recruitment and clinical assessment of rheumatology patients. $\mathrm{MC}$ gave final approval for the manuscript. All authors read and approved the final manuscript.

\section{Additional material}

\section{Additional file 1}

Acquisition of bidimensional echocardiographic images during treadmill exercise: preparing alignment of continuous wave Doppler with the tricuspid regurgitant jet. The data provided represent the alignment of continuous wave Doppler with the tricuspid regurgitant jet, during acquisition of bidimensional echocardiographic images during treadmill exercise.

Click here for file

[http://www.biomedcentral.com/content/supplementary/14767120-6-51-S1.wmv]

\section{Additional file 2}

Continuous wave Doppler signal of the tricuspid regurgitant jet, obtained during treadmill exercise. The data provided represent the continuous wave Doppler signal of the tricuspid regurgitant jet, obtained during treadmill exercise.

Click here for file

[http://www.biomedcentral.com/content/supplementary/14767120-6-51-S2.wmv]

\section{Additional file 3}

Injection of the agitated saline with blood contrast, filling the right cavities. The data provided represent the right cavities filled with agitated saline with blood contrast after injection.

Click here for file

[http://www.biomedcentral.com/content/supplementary/14767120-6-51-S3.avi]

\section{Additional file 4}

Ventricular septal defect, demonstrated by the injection of contrast in one of our patients. The data provided represent the ventricular septal defect, demonstrated by the injection of contrast in one of our patients. Click here for file

[http://www.biomedcentral.com/content/supplementary/14767120-6-51-S4.avi]

\section{References}

I. Yock PG, Popp RL: Noninvasive estimation of right ventricular systolic pressure by Doppler ultrasound in patients with tricuspid regurgitation. Circulation 1984, 70(4):657-62.

2. Berger M, Haimowitz A, Van Tosh A, Berdoff RL, Goldberg E: Quantitative assessment of pulmonary hypertension in patients with tricuspid regurgitation using continuous wave Doppler ultrasound. I Am Coll Cardiol 1985, 6(2):359-65.

3. Currie PJ, Seward JB, Chan KL, Fyfe DA, Hagler DJ, Mair DD, Reeder GS, Nishimura RA, Tajik AJ: Continuous wave Doppler determination of right ventricular pressure: a simultaneous Dopplercatheterization study in 127 patients. J Am Coll Cardiol 1985, 6(4):750-6.

4. Vazquez de Prada JA, Ruano J, Martin-Duran R, Larman M, Zueco J, Ortiz de Murua JA, Torres A, Figueroa A: Noninvasive determination of pulmonary arterial systolic pressure by continuous wave Doppler. Int I Cardiol I987, I6(2): 177-84.

5. Dubourg O, Delorme G, Jondeau G, Chikli F, Clavier H, Valtier B, Terdjman M, Beauchet A, Bourdarias JP: Simultaneous measurement of systolic pulmonary artery pressure by catheterization and contrast enhancement doppler echocardiography. Arch Mal Coeur Vaiss 1993, 86(12): I72I-7.

6. Tokushima T, Utsunomiya T, Yoshida K, Ogawa T, Kido K, Ohtsubo Y, Ryu T, Ogata T, Tsuji S, Matsuo S: Estimation of the systolic pulmonary arterial pressure using contrast-enhanced continuous-wave Doppler in patients with trivial tricuspid regurgitation. Jpn Heart J 1999, 40(3):3 I I-20.

7. Jeon DS, Luo H, Iwami T, Miyamoto T, Brasch AV, Mirocha J, Naqvi TZ, Siegel RJ: The usefulness of a $10 \%$ air- $10 \%$ blood $-80 \%$ saline mixture for contrast echocardiography: Doppler measurement of pulmonary artery systolic pressure. J Am Coll Cardiol 39(I): 124-9. 2002 Jan 2

8. Jeon DS, Luo H, Brasch AV, Nagai T, Miyamoto T, Mohsenifar Z, Siegel RJ: Superiority of $10 \%$ air- $10 \%$ blood-saline mixture for measuring the velocity of tricuspid regurgitation in patients with severe emphysema. JAm Soc Echocardiogr 2003, 16(8):867-70.

9. Marwick TH: Application of stress echocardiography to the evaluation of non-coronary heart disease. Eur J Echocardiography 2000, I:17I-179. 
10. Bossone E, Bodini BD, Mazza A, Allegra L: Pulmonary arterial hypertension: the key role of echocardiography. Chest 2005, I27(5):1836-43.

II. Vachiéry JL, Pavalescu A: Exercise echocardiography in pulmonary hypertension. Eur Heart J Suppl 2007, 9:H48-H53. doi:10.1093/eurheartj/sum022

12. Sicari R, Nihoyannopoulos P, Evangelista A, Kasprzak J, Lancellotti P, Poldermans D, Voigt JU, Zamorano JL, European Association of Echocardiography: Stress echocardiography expert consensus statement: European Association of Echocardiography (EAE) (a registered branch of the ESC). Eur J Echocardiogr 2008, 9:4I5-37.

13. Galié N, et al:: Guidelines on Diagnosis and Treatment of Pulmonary Arterial Hypertension. Eur Heart J 2004, 25:2243-2278.

Publish with Bio Med Central and every scientist can read your work free of charge

"BioMed Central will be the most significant development for disseminating the results of biomedical research in our lifetime. "

Sir Paul Nurse, Cancer Research UK

Your research papers will be:

- available free of charge to the entire biomedical community

- peer reviewed and published immediately upon acceptance

- cited in PubMed and archived on PubMed Central

- yours - you keep the copyright 